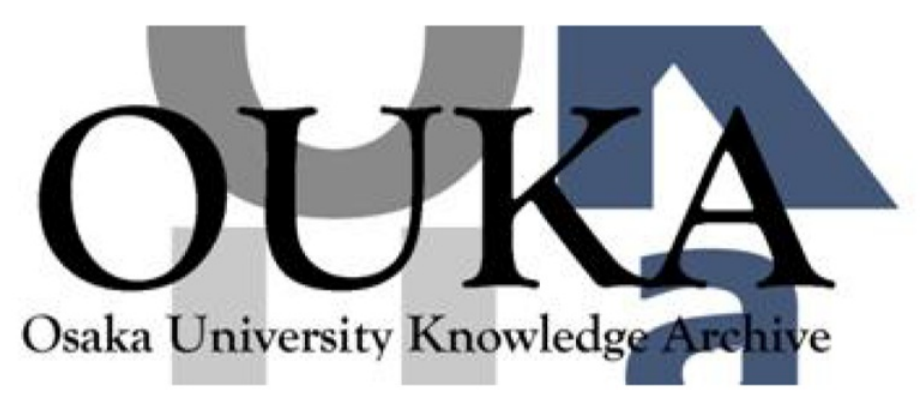

\begin{tabular}{|c|c|}
\hline Title & $\begin{array}{l}\text { Nano-plate biosensor array using ultrafast heat } \\
\text { transport through proteins }\end{array}$ \\
\hline Author(s) & $\begin{array}{l}\text { Ogi, Hirotsugu; Iwagami, Shigeru; Nagakubo, } \\
\text { Akira et al. }\end{array}$ \\
\hline Citation & $\begin{array}{l}\text { Sensors and Actuators, B: Chemical. } 278 \text { p. 15- } \\
\text { p. } 20\end{array}$ \\
\hline Issue Date & $2018-09-17$ \\
\hline oaire:version & AM \\
\hline URL & https://hdl. handle. net/11094/84197 \\
\hline rights & $\begin{array}{l}\text { - } 2018 \text { Elsevier B.V. This manuscript version is } \\
\text { made avai lable under the Creative Commons } \\
\text { Attribution-NonCommercial-NoDerivatives } 4.0 \\
\text { International License. }\end{array}$ \\
\hline Note & \\
\hline
\end{tabular}

Osaka University Knowledge Archive : OUKA

https://ir. Library. osaka-u. ac. jp/

0saka University 


\title{
Nano-Plate Biosensor Array Using Ultrafast Heat Transport through Proteins
}

\author{
Hirotsugu Ogi ${ }^{a}$, Shigeru Iwagami ${ }^{b}$, Akira Nagakubo $^{a}$, Takuya Taniguchi $^{c}$, \\ Teruo Ono ${ }^{d}$ \\ ${ }^{a}$ Graduate School of Engineering, Osaka University \\ Yamadaoka 2-1, Suita, Osaka 565-0871, Japan \\ ${ }^{b}$ Graduate School of Engineering Science, Osaka University \\ Machikaneyama 1-3, Toyonaka, Osaka 560-8531, Japan \\ ${ }^{c}$ Technische Universität München \\ ${ }^{d}$ Institute for Chemical Research, Kyoto University \\ Gokasho, Uji, Kyoto, 611-0011, Japan
}

\begin{abstract}
A novel label-free biosensor array is proposed, which uses the ultrafast heat transport from a metallic nano-plate into surrounding solution across proteins captured on the nano-plate. Using electron-beam lithography, 2,500 gold nano-plates of $30 \mathrm{~nm}$ thick with $5 \times 5 \mu \mathrm{m}^{2}$ area were fabricated on a fused silica substrate, on which receptor proteins were immobilized. Thermal phonons were induced on the back surface of a single nano-plate by focusing ultrafast light pulses from the substrate side, which propagated toward surrounding solution through surface proteins. Because the ultrafast heat-transport behavior, which is completed within $\sim 1 \mathrm{~ns}$, is affected by existence of target proteins on the nano-plate, each nano-plate works as a single label-free biosensor. A theoretical calculation was made for predicting the cooling-down behavior of the nano-plate after an impulsive heating, and a pump-probe optics was developed for monitoring the ultrafast temperature change after excitation with a femtosecond fiber laser. We then confirm that each nano-plate acts as a single label-free biosensor by performing antigen-antibody binding reactions, indicating that a large scale number of multichannel measurement is made possible with this phenomenon.
\end{abstract}

Keywords: label-free biosensor, pump-probe laser measurement, thermoreflectance measurement, MEMS, multichannel 


\section{Introduction}

The label-free biosensor allows a short-time detection of target biomolecules because it is free from extra incubation and washing procedures. It also allows a real-time monitoring of binding and dissociation reactions between biomolecules, providing the binding affinity between them quantitatively. Thus, the label-free biosensor is promising in diagnosis and drug-development processes. An unsolved assignment is development of a large-scale multichannel label-free biosensors. There are two representative label-free biosensors: One is the surface-plasmon-resonance (SPR) biosensor $[1,2,3]$ and the other is the quartz-crystal-microbalance (QCM) biosensor $[4,5,6]$. Intensive efforts have been made for developing multichannel SRP [7, 8, 9] and multichannel QCM $[10,11,12]$ sensors. Other label-free multichannel biosensors are recently reported, including the nanoplasmonic biosensors [13], electrochemical biosensors [14], and optical-waveguide-based biosensors [15]. However, the number of channels has been typically smaller than 50, and the single sensor area is larger than $\sim 50 \times 50 \mu \mathrm{m}^{2}$. Thus, a drastic increase in the channel number with smaller sensing areas is required in the label-free-biosensor field.

In this study, we propose a novel label-free biosensor, that uses ultrafast heat propagation from a metallic nano-plate into surrounding solution across biomolecules immobilized and captured on the nano-plate surface. Because metallic materials exhibit much higher thermal diffusivities than that of water, after it is heated, it remains warm without the protein layer on it. However, when a dense protein layer exits between the surrounding solution and the metallic nano-plate, the heat-transfer behavior into the solution region should be accelerated because thermal diffusivity of proteins are expected to be higher than that of water; the protein layer works as a coupling material for heat transfer. The cooling-down behavior of the metallic nano-plate after heated will then highly depend on the presence of the protein layer. It is, therefore, possible to develop a label-free biosensor using this phenomenon by measuring the nano-plate temperature after heating. A label-free biosensor using the heat-transfer principle has been reported [16], but it is not adopted for a large-scale multichannel biosensor application.

There are some distinguished advantages in this biosensor. First, a single sensor channel can be significantly minimized, because heating the nano-plate and detecting its temperature are accomplished by focusing light pulses onto 
$\sim 1 \mu \mathrm{m}^{2}$ area. Second, the number of channels can be drastically increased by fabricating the nano-plate arrays on a substrate with a MEMS process. Third, a short-time measurement is made possible, because the heat transfer from a nano-plate into surrounding water complete within $\sim 1 \mathrm{~ns}$.

First, we perform a theoretical analysis for evaluating the effect of growth of the protein layer on the nano-plate on the cooling-down behavior of the nano-plate after heated. The one-dimensional heat-transfer model for multilayer is established, and the fast temperature change after the excitation is calculated using the Fourier transform method.

We then demonstrate the availability of the proposed biosensor: We develop an optics for measuring the ultrafast heat transport behavior with a femtosecond fiber laser. Using the electron-beam-lithography method, we fabricated 2,500 gold nano-plates with $5 \mu \mathrm{m} \times 5 \mu \mathrm{m}$ area of 30-nm thick on a fused-silica substrate and performed the real-time monitoring of antigenantibody binding reactions on a single nano-plate. Our measurements show agreement with the theoretical analysis, and we confirm that this new type of biosensor array works as multichannel label-free biosensors.

\section{Theoretical Analysis}

Figure 1(a) illustrates the cross-section view near a single nano-plate, consisting of the glass substrate, Au nano-plate, proteins on it, and surrounding solution. Because the in-plane dimension of Au nano-plate $(5 \mu \mathrm{m})$ is much larger than its thickness $(30 \mathrm{~nm})$ and heating by a light pulse is made on the entire back surface of the nano-plate, the thermal transport occurs nearly with one-dimensional manner. The thermal diffusivity of the glass substrate is much smaller than that of $\mathrm{Au}$ by a factor of $\sim 0.005$, and the bottom face of the Au nano-plate can be considered as an adiabatic face. We therefore simplify the heat transport phenomenon with the three layer model as shown in Fig. 1(b), where the protein layer is assumed to be a continuous and homogenous layer; the progress in the binding reaction is regarded as the increase in the effective thickness, $d_{p}$, of the protein layer.

The one-dimensional heat-transfer behavior in homogenous multilayer system has been analyzed previously $[17,18]$, and we follow their approaches. We briefly show the procedure we made. The temperature change $T(t, z)$ in $i$ th layer obeys the heat equation:

$$
\frac{\partial T}{\partial t}=\frac{\kappa_{i}}{C_{i} \rho_{i}} \cdot \frac{\partial^{2} T}{\partial z^{2}} \equiv \alpha_{i} \frac{\partial^{2} T}{\partial z^{2}} .
$$


Here, $t, \kappa, C$, and $\rho$ denote time, thermal conductivity, specific heat, and mass density, respectively. $\alpha=\kappa / C \rho$ is thermal diffusivity. $z$ is the distance measured from the back surface of the Au nano-plate (Fig. 1(b)). The subscript $i$ indicates a quantity of the $i$ th layer. Adopting the Fourier transform for $t$ with $\omega$, we obtain

$$
\frac{\partial^{2} \hat{T}(\omega, z)}{\partial z^{2}}=\frac{j \omega}{\alpha_{i}} \hat{T}(\omega, z)
$$

The general solution takes the form:

$$
\hat{T}(\omega, z)=C_{1}(\omega) e^{q z}+C_{2}(\omega) e^{-q z},
$$

with $q=\sqrt{j \omega / \alpha}$. Using the boundary conditions at lower and upper surfaces of the $i$ th layer (Fig. 2), we can eliminate the coefficients $C_{1}(\omega)$ and $C_{2}(\omega)$ and obtain a relationship between temperature $\hat{T}$ and heat flux $\hat{f}$ at the lower (input) and upper (output) surfaces of the $i$ th layer

$$
\left(\begin{array}{c}
\hat{T}_{i}^{(\text {out })}(\omega) \\
\hat{f}_{i}^{(\text {out })}(\omega)
\end{array}\right)=\left(\begin{array}{cc}
\cosh \left(q_{i} d_{i}\right) & -\frac{1}{\kappa_{i} q_{i}} \sinh \left(q_{i} d_{i}\right) \\
-\kappa_{i} q_{i} \sinh \left(q_{i} d_{i}\right) & \cosh \left(q_{i} d_{i}\right)
\end{array}\right)\left(\begin{array}{c}
\hat{T}_{i}^{(i n)}(\omega) \\
\hat{f}_{i}^{(i n)}(\omega)
\end{array}\right) \equiv \mathbf{M}_{i}\left(\begin{array}{c}
\hat{T}_{i}^{(i n)}(\omega) \\
\hat{f}_{i}^{(i n)}(\omega)
\end{array}\right)
$$

with the heat-transfer matrix $\mathbf{M}_{i}$ Analogous analysis allows derivation of the relationship between the temperature and heat flux at the top and bottom surfaces of the multilayer system by multiplying the transfer matrices of individual layers:

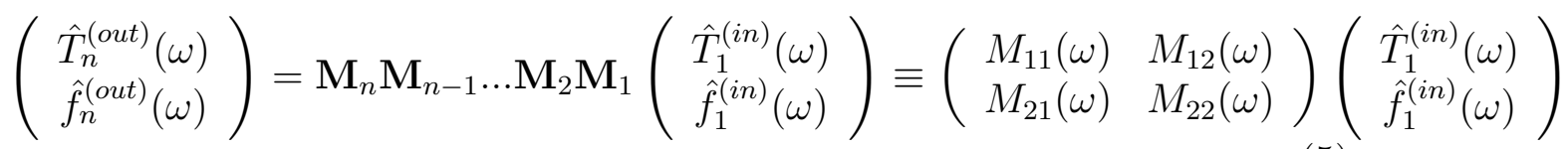

Because we heat the lowest layer by irradiating the bottom surface with a light pulse with duration $a$, the heat flux $f_{1}(t)$ is expected to become $f_{1}(t)=H(t) e^{-a t}$, where $H(t)$ denotes the Heaviside function. Thus, we obtain

$$
\hat{f}_{1}^{(i n)}(\omega)=\frac{1}{a+j \omega} .
$$

Furthermore, because the water layer (top layer) is sufficiently thicker than the protein and gold layers, the heat flux at the top surface should be zero, yielding the relationship

$$
\hat{T}_{1}^{(i n)}(\omega)=-\frac{M_{22}(\omega)}{M_{21}(\omega)} \hat{f}_{1}^{(i n)}(\omega)
$$


We then obtain the temperature change at the bottom surface, which is measurable, with the Fourier transformation

$$
T_{1}^{(i n)}(t)=\int_{-\infty}^{\infty}\left[-\frac{M_{22}(\omega)}{M_{21}(\omega)(a+j \omega)}\right] e^{j \omega t} d \omega
$$

\section{Experimental Section}

\subsection{Pump-probe optics for thermoreflectance measurement}

Figure 3(a) shows the optics developed for this study. We used a femtosecond fiber laser (Fianium, FP-1060-1) with $\sim 150$ fs pulse width, 50 $\mathrm{MHz}$ repetition frequency, and $1064 \mathrm{~nm}$ wavelength. The light pulse was frequency-doubled with the second-harmonic-generator (SHG) crystal, and split into pump and probe pulses with the polarized beam splitter (PBS) after passing through a $\lambda / 2$ plate, which controls the polarization angle and the split ratio between the pump and probe lights. The pump light was amplitude-modulated by an acousto-optic crystal with $1 \mathrm{MHz}$ modulation frequency and, after passing on the light path involving the corner reflector, it was focused on back face of a single nano-plate with a 100-magnification objective lens to transiently heat the nano-plate. The probe light pulse was focused on the nano-plate back surface by the same objective lens to measure the temperature there via the photo-thermal (thermoreflectance) effect, where the reflectivity change of the probe light is proportional to temperature. A part of the probe light was acquired by a balanced photo detector as a reference using a beam splitter before irradiation of the nano-plate, and the reflected probe light from the nano-plate was received by the balance detector. The reference-subtracted signal was fed to a lock-in amplifier to extract the modulation-frequency component. The energy of the pump and probe light pulses was about $20 \mathrm{pJ}$. By changing the path length of the pumping light pulse by moving the corner reflector, the delay time of the probe light pulse to the pump light pulse at the nano-plate surface was controlled in a $\sim 100$ fs time resolution.

\subsection{Surface modification of gold nano-plates}

We fabricated 2,500 Au nano-plates in a $0.5 \mathrm{~mm} \times 0.5 \mathrm{~mm}$ area on a fusedsilica substrate of $0.3 \mathrm{~mm}$ thick using electron-beam lithography method (Fig. $4(\mathrm{a}))[19,20]$. A single nano-plate shows a square shape with $5 \mu \mathrm{m}$ side as shown in Fig. 4(b), and its thickness is $30 \mathrm{~nm}$ ( $25 \mathrm{~nm} \mathrm{Au}$ on $5 \mathrm{~nm} \mathrm{Cr}$ ). 
In this study, we immobilized four different proteins on different areas with a micropipette as follows: We first washed the nano-plate array with piranha solution $\left(98 \% \mathrm{H}_{2} \mathrm{SO}_{4}: 33 \% \mathrm{H}_{2} \mathrm{O}_{2}=4: 1\right)$. After rinsing it with ultrapure water, its was cleaned with a UV ozone cleaner. The chip was then immersed in $10 \mathrm{mM}$ 10-carboxy-1-decanethiol (Dojindo, C385) solution for $18 \mathrm{~h}$ at 4 ${ }^{\circ} \mathrm{C}$. After rinsing it with ultrapure water, the nano-plate surfaces were activated by $100 \mathrm{mM}$ EDC(1-ethyl-3-(3-dimethylaminopropyl)carbodiimide, hydrochloride; Dojindo, W001) solution and 100 mM NHS (N-Hydroxysuccinimide; Wako, 089-04032) solution for $1 \mathrm{~h}$. After rinsing it with ultrapure water, four droplets of four receptor-protein solutions shown below were deposited on the first, second, third, and fourth quadrants in the nano-plate array for immobilizing four different receptor proteins on the different areas. After washing with phosphate-buffered-saline (PBS) solution, the nano-plate array was immersed in $10 \mathrm{mg} / \mathrm{ml}$ bovine-serum-albumin (BSA; Sigma, A3059$10 \mathrm{G}$ ) solution for blocking remaining activated sites. After washing it with PBS solution, the sensor chip was set in the sensor cell as shown in Fig. 3(b). The receptor-protein solutions were $200 \mu \mathrm{g} / \mathrm{ml}$ anti rabbit IgG antibody (Anti-rIgG; Arista Biilogicals, Inc. ABGAR-0500) solution, $200 \mu \mathrm{g} / \mathrm{ml}$ anti human IgG antibody (Anti-hIgG; Invitrogen, ABIGG-0500) solution, $200 \mu \mathrm{g} / \mathrm{ml}$ protein A (Invitrogen, 10-1100) solution, and the BSA solution. The target protein was rabbit IgG (rIgG) (Invitrogen, 21616237) and its solution was injected by a piezo-micropump with a flow rate of $100 \mu \mathrm{l} / \mathrm{min}$, which was carried along the nano-plate surfaces as shown in Fig. 3(b).

\section{Results and Discussion}

\subsection{Theoretical calculation}

Figure 5 compares the reflectivity change $\Delta R$ of the probe light with theoretical calculation (Eq. (8)) for the temperature change on the back surface of $30-\mathrm{nm}$ gold layer without protein layer. The theoretical calculation principally agrees with the measurement: The transient raise and drop in temperature after excitation, and subsequent gradual cooling-down process are consistently explained by the theoretical calculation, indicating that influence of protein layer on the cooling-down behavior can be evaluated with the theoretical calculation.

While thermal properties of proteins are highly ambiguous, several researchers estimated protein thermal conductivity to be of the order of 0.1 $\mathrm{W} / \mathrm{Km}[21,22,23]$, and we here assumed it to be $0.2 \mathrm{~W} / \mathrm{Km}$. Also, we 
used $3.5 \times 10^{-7} \mathrm{~m}^{2} / \mathrm{s}$ for thermal diffusivity of the protein layer, referring prediction by $\mathrm{Yu} \&$ Leitner [21]. Figure 6(a) shows the heating-cooling down behavior calculated for various protein-layer thicknesses $d_{p}$ defined in Fig. 1(b). We find three notable characteristics: First, the cooling-down process is enhanced as the protein layer becomes thicker, indicating that the protein layer acts as a coupling material for heat flow from nano-plate to solution. Second, the temperature change depends on the time after the excitation. At a later stage (for example at $100 \mathrm{ps}$ ), the temperature change will not be monotonous to the protein layer thickness. Third, the cooling-down behavior is sensitive to the thickness change when $d_{p}<\sim 50 \mathrm{~nm}$, but it becomes insensitive to that for $d_{p}>\sim 70 \mathrm{~nm}$ as shown in Fig. $6(\mathrm{~b})$ : A thick enough protein layer will work as an insulator material rather than the coupling material for heat transfer. Because height of an IgG molecule is about $10 \mathrm{~nm}$ [24], the protein layer thickness after binding reaction will be typically thinner than $\sim 30 \mathrm{~nm}$, and the high sensitivity of the heat transfer phenomenon to the change in the protein thickness in a region of $d_{p}<\sim 50 \mathrm{~nm}$ is promising as a label-free biosensor.

\subsection{Detection of antigen-antibody binding reactions}

Figure 7 shows the reflectivity changes (i.e. temperature changes) on different nano-plates on which anti-rIgG antibody or anti-hIgG antibody was immobilized. We injected 6-nM rIgG solution in PBS and obtained them after $30 \mathrm{~min}$. We focused the light pulses on a single nano-plate as shown in Fig. 4(c). Three independent measurements on three different nanoplates are shown in the figure, indicating sufficient reproducibility. On the positive nano-plates (anti-rIgG channels), significant temperature decreases were observed, whereas on the negative nano-plates (anti-hIgG channels), the responses were close to those observed in the injection of the PBS solution (control). Thus, these experiments demonstrate that the tiny dot $(5 \mu \mathrm{m}$ square) works as one label-free biosensor.

For evaluating the cross-talk effect, we performed the reflectivity-change measurement on a neighbor nano-plate by irradiating a nano-plate with the pump light and applying the probe light on the neighbor (different) nanoplate. As shown in Fig. 7 with a broken line, no significant reflectivity change is observed. This result is reasonable because the heat transfer phenomenon occurs in very thin $(\sim 30 \mathrm{~nm})$ nano-plate, and the distance between nano-plates $(5 \mu \mathrm{m})$ is much larger than the nano-plate thickness. Therefore, 
the cross-talk effect is negligible in this measurement, and we expect that independent assay is made possible on each nano-plate.

We sometimes observed high-frequency oscillations after the excitation as seen in Fig. 7; its frequency is about $310 \mathrm{GHz}$. There are three possible causes for the reflectivity oscillation. (1) Mechanical oscillation of nano-plate [25]. The gold nano-plate could be resonated by the pump probe light because of the transient thermal expansion and shrink. The highest resonance frequency corresponds to the through-thickness resonance frequency, which is estimated to be about $79 \mathrm{GHz}$. This is significantly lower than the observed frequency, and this mechanism should not be the cause. (2) Brillouin oscillation from the glass substrate [26], which is caused by interference between the reflected probe light at the nano-plate back surface and refracted probe light by the acoustic wave propagating in the glass susurrate. Its frequency is, however, estimated to be about $45 \mathrm{GHz}$, which is again lower. (3) Phonon vibration of surface proteins. This could be observed because the phonon pulse is generated by the pump light pulse, and it could cause vibrations of proteins. However, we have currently no convincing confirmation about this, and identification of the high-frequency oscillation will be our future study.

Figure 8(a) shows time changes of the reflectivity (or temperature) at 50 ps after excitation on four different nano-plate channels, on which anti-rIgG antibody, protein A, anti-hIgG antibody, and BSA are immobilized, during flowing the 6-nM rIgG solution. On the negative channels (BSA and antihIgG antibody channels), insignificant temperature decreases are observed, whereas on the positive channels (anti-rIgG antibody and protein A channels), temperature largely decreases after the arrival of the analyte. Figure 8(b) shows the amount of the reflectivity change at 2000 s from the injection, clearly demonstrating the high selectivity of the proposed biosensor.

Figure 9(a) shows the temperature change behaviors of a nano-plate, on which the Anti-rIgG antibody was immobilized, after rIgG solutions with various concentrations were injected. The sensor response increases as the target-protein concentration increases, but it is saturated in higher concentration region as shown in Fig. 9(b). This nonlinear behavior is expected form the theoretical calculation (Fig. 6); a thick protein layer will restrict the temperature change of the nano-plate because it works as an insulator. 


\section{CONCLUSION}

The ultrafast heat-transport phenomenon from a gold nano-plate into solutions across adsorbed proteins was theoretically and experimentally studied. The theoretical calculation reveals that the cooling-down behavior of the nano-plate after an impulsive heating is significantly affected by the growth of the protein layer on the nano-plate when the protein layer is thinner than $\sim 70 \mathrm{~nm}$, indicating that monitoring temperature on the nano-plate after heating allows development of a label-free biosensor. We fabricated 2,500 channel gold nano-plates on a fused-glass substrate; a single nanoplate is a square with $5 \mu \mathrm{m}$ sides and $30-\mathrm{nm}$ thickness, and then performed the transient-heating and subsequent temperature-monitoring experiments with originally developed optics with a femtosecond fiber laser. We then demonstrated that a single nano-plate works as a label-free biosensor using the antigen-antibody binding reaction. Because it is technically possible to immobilize 2,500 different receptor proteins on individual nano-plates using an inkjet printing technique, a large-scale multichannel label-free biosensor will be developed with the proposed method. Currently, the detection limit is about $7 \mathrm{pM}$ and a liner response appears between 6.7 and $670 \mathrm{pM}$. These properties will be controlled by changing the thickness and material of the nano-plate. Although it is not easy to perform the 2,500 channel bioassays simultaneously, using a charge-coupled-device (CCD) or complementary metaloxide-semiconductor (CMOS) detectors will overcome this difficulty, which will be our future study. 
[1] B. Liedberg, C. Nylander, I. Lunström, I., Surface plasmon resonance for gas detection and biosensing, Sens. Actuators 4 (1983) 299-304.

[2] D. Nedelkov, Development of surface plasmon resonance mass spectrometry array platform, Anal. Chem. 76 (2007) 5987-5990.

[3] X.D. Hoa, A.G. Kirk, M. Tabrizian, Towards integrated and sensitive surface plasmon resonance biosensors: A review of recent progress, Biosens. Bioelectron. 23 (2007) 151-160.

[4] H. Muramatsu, M.D. Dicks, E. Tamiya, I. Karube, Piezoelectric crystal biosensor modified with protein A for determination of immunoglobulins, Anal. Chem. 59 (1987) 2760-2763.

[5] Y. Liu, X. Yu, R. Zhao, D. Shangguan, Z. Bo, G. Liu, Quartz crystal biosensor for real-time monitoring of molecular recognition between protein and small molecular medicinal agents, Biosens. Bioelectron. 19 (2003) 9-19.

[6] H. Ogi, K. Motohisa, T. Matsumoto, K. Hatanaka, M. Hirao, Isolated electrodeless high-frequency quartz crystal microbalance for immunosensors, Anal. Chem. 78 (2006) 6903-6909.

[7] J. Homola, H.B. Lu, G.G. Nenningera, J. Dostálek, S.S. Yee, A novel multichannel surface plasmon resonance biosensor, Sens. Actuat. B 76 (2001) 403-410.

[8] R. Otupiri, E.K. Akowuah, S. Haxha, Multi-channel SPR biosensor based on PCF for multi-analyte sensing applications, Opt. Exp. 23 (2015) 1571615727.

[9] Y. Liu, S. Chen, Q. Liu, J.F. Masson, W. Peng, Compact multi-channel surface plasmon resonance sensor for real-time multi-analyte biosensing, Opt. Exp. 23 (2015) 20540-20548.

[10] T. Abe and M. Esashi, One-chip multichannel quartz crystal microbalance (QCM) fabricated by deep RIE, Sens. Actuat. A 82 (2000) 139-143.

[11] X. Jin, Y. Huang, A. Mason, X. Zeng, Multichannel monolithic quartz crystal microbalance gas sensor array, Anal. Chem. 81 (2009) 595-603. 
[12] H. Ogi, H. Nagai, Y. Fukunishi, T. Yanagida, M. Hirao, M. Nishiyama, Multichannel wireless-electrodeless quartz-crystal microbalance immunosensor, Anal. Chem. 82 (2010) 3957-3962.

[13] M. Soler, A. Belushkin, A. Cavallini, C. Kebbi-Beghdadi, G. Greub, H. Altug, Multiplexed nanoplasmonic biosensor for one-step simultaneous detection of Chlamydia trachomatis and Neisseria gonorrhoeae in urine, Biosens. Bioelectron. 94 (2017) 560-567.

[14] J. Bruchmann, K. Sachsenheimer, B.E. Rapp, T. Schwartz, Multichannel microfluidic biosensor platform applied for online monitoring and screening of biofilm formation and activity, PLoS ONE 10 (2015) e0117300.

[15] J. Wang, Z. Yao, T. Lei, A.W. Poon, Silicon coupled-resonator opticalwaveguide-based biosensors using light-scattering pattern recognition with pixelized mode-field-intensity distributions, Sci. Rep. 4 (2014) 7528.

[16] M. Peeters, B. van Grinsven, T.J. Cleij, K.L. Jiménez-Monroy, P. Cornelis, E. Pérez-Ruiz, G. Wackers, T. Thoelen, W.D. Ceuninck, J. Lammertyn, P. Wagner, Label-free protein detection based on the heat-transfer Method - A case study with the peanut allergen ara h 1 and aptamer-based synthetic receptors, ACS Appl. Mater. Interfaces 7 (2015) 10316-10323.

[17] H.S. Carslaw, J.C. Jaeger, Conduction of Heat in Solids (Oxford University Press, New York, 1959).

[18] A.J. Schmidt, X. Chen, G. Chen, Pulse accumulation, radial heat conduction, and anisotropic thermal conductivity in pump-probe transient thermoreflectance, Rev. Sci. Instrum. 79 (2008) 114902.

[19] H. Ogi, A. Yamamoto, K. Kondou, K. Nakano, K. Morita, N. Nakamura, T. Ono, M. Hirao, Significant softening of copper nanowires during electromigration studied by picosecond ultrasound spectroscopy, Phys. Rev. B 82 (2010) 155436.

[20] H. Ogi, S. Masuda, A. Nagakubo, N. Nakamura, M. Hirao, K. Kondou, T. Ono, Impact of interface stiffness in surface-wave resonances on nanostrip-attached substrates, Phys. Rev. B 93 (2016) 024112.

[21] X. Yu, M.D. Leitner, Vibrational energy transfer and heat conduction in a protein, J. Phys. Chem. B 107 (2003) 1698-1707. 
[22] A. Lervik, F. Bresme, S. Kjelstrup, D. Bedeauxbc, J.M. Rubi, Heat transfer in protein-water Interfaces, Phys. Chem. Chem. Phys. 12 (2010) 1610-1617.

[23] B.M. Foley, C.S. Gorham, J.C. Duda, R. Cheaito, C.J. Szwejkowski, C. Constantin, B. Kaehr, P.E. Hopkins, Protein thermal conductivity measured in the solid state reveals anharmonic interactions of vibrations in a fractal structure, J. Phys. Chem. Lett. 5 (2014) 1077-1082.

[24] V.R. Sarma, E.W. Silverton, D.R. Davies, W.D. Terry, The threedimensional structure at 6 A resolution of a human $\gamma \mathrm{G} 1$ immunoglobulin molecule, J. Biol. Chem. 10 (1971) 3753-3759.

[25] H. Ogi, M. Fujii, N. Nakamura, T. Yasui, M. Hirao, Stiffened ultrathin Pt films confirmed by acoustic-phonon resonances, Phys. Rev. Lett. 98 (2007) 195503.

[26] H. Ogi, T. Shagawa, N. Nakamura, M. Hirao, H. Odaka, N. Kihara, Elastic constant and Brillouin oscillations in sputtered vitreous $\mathrm{SiO}_{2}$ thin films, Phys. Rev. B 78 (2008) 134204. 


\section{Figure Caption}

Fig. 1 (a)Schematic of cross-section of gold nano-plate on a fused-glass substrate, on which proteins are adsorbed. (b)Three-layer model for measurement configuration.

Fig. 2 Multilayer model for heat transfer phenomenon from the bottom surface of the lowest layer to upper direction.

Fig. 3 (a)Optics developed for monitoring the temperature on the nanoplate back surface after the transient heating. A femtosecond fiber laser was used for this. (b)Cross-section schematic view of the sensor sell, where the fused-silica plate with many gold nano-plates is set. The solution flows along the nano-plate surfaces and the light pulses enter a small window of the sensor cell.

Fig. 4 (a)A photograph for the nano-plate array chip. (b)An enlarged view obtained by a scanning electron microscope. (c)Focused light pulse for heating the gold nano-plate, achieving the label-free bioassay on a single nano-plate.

Fig. 5 (a)Measured reflectivity change of the single gold nano-plate after heating with a 150-fs light pulse. (b)Calculated back-surface temperature change of the gold layer after the impulsive heating with $150 \mathrm{fs}$ without protein layer. The injected laser pulse power was $20 \mathrm{~mW}$ both in experiment and calculation.

Fig. 6 (a)Analytical calculation of the change of the back-surface temperature of the gold layer after the 150-fs impulsive heating. (b)Relationship between the protein layer thickness and the back-surface temperature at 50 ps after heating.

Fig. 7 Reflectivity change (i.e. temperature change) after heating of different nano-plates after flowing 6.7-nM rabbit-IgG solution for $30 \mathrm{~min}$. 
Red and black lines indicate measurements for nano-plates on which anti-rabbit IgG antibody (positive plate) and anti-human IgG antibody (negative plate) are immobilized, respectively. Blue lines indicate responses for the positive nano-plates when phosphate buffered saline (PBS) solution was injected (control). Three independent measurements for three different nano-plates are shown for each case. The broken line is the response when the probe light irradiated the neighbor (different) nano-plate.

Fig. 8 (a)Reflectivity change (i.e. temperature change) at 50 ps after the impulsive heating on four different nano-plates, on which BSA (negative), anti-hIgG antibody (negative), protein A (positive), and antirIgG antibody (positive) are immobilized, during the flow of 6.7-nM rabbit-IgG solution. (b) Reflectivity change (temperature change) at $2000 \mathrm{~s}$ for the four channels.

Fig. 9 (a)Temperature behaviors of a single nano-plate, on which anti-rIgG antibody was immobilized, after injection of rIgG solutions with concentrations between $6.7 \mathrm{pM}$ and $6.7 \mathrm{nM}$. (b)Relationship between the concentration of the injected rIgG and the sensor response at $2000 \mathrm{~s}$. 


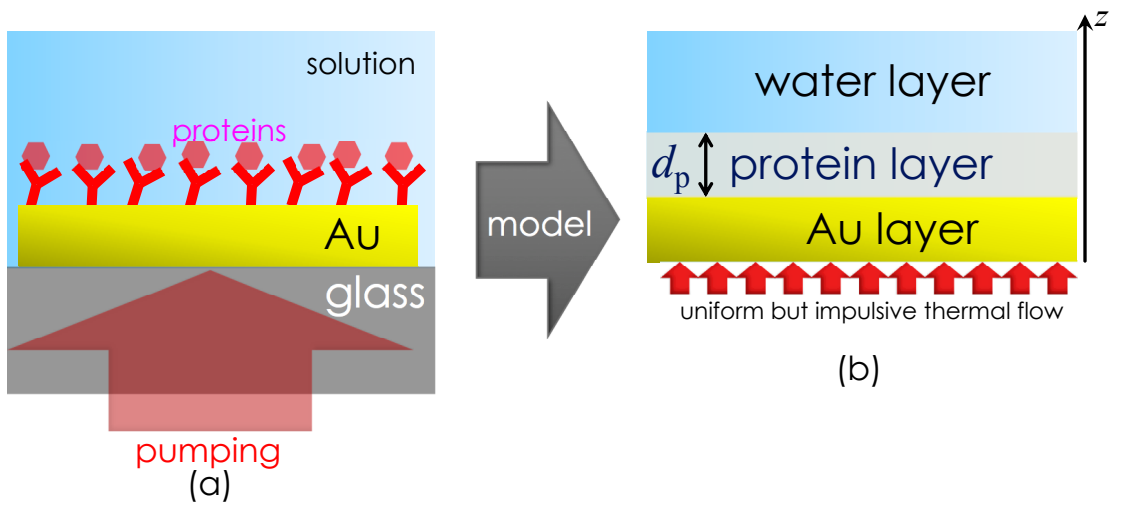

Figure 1: 


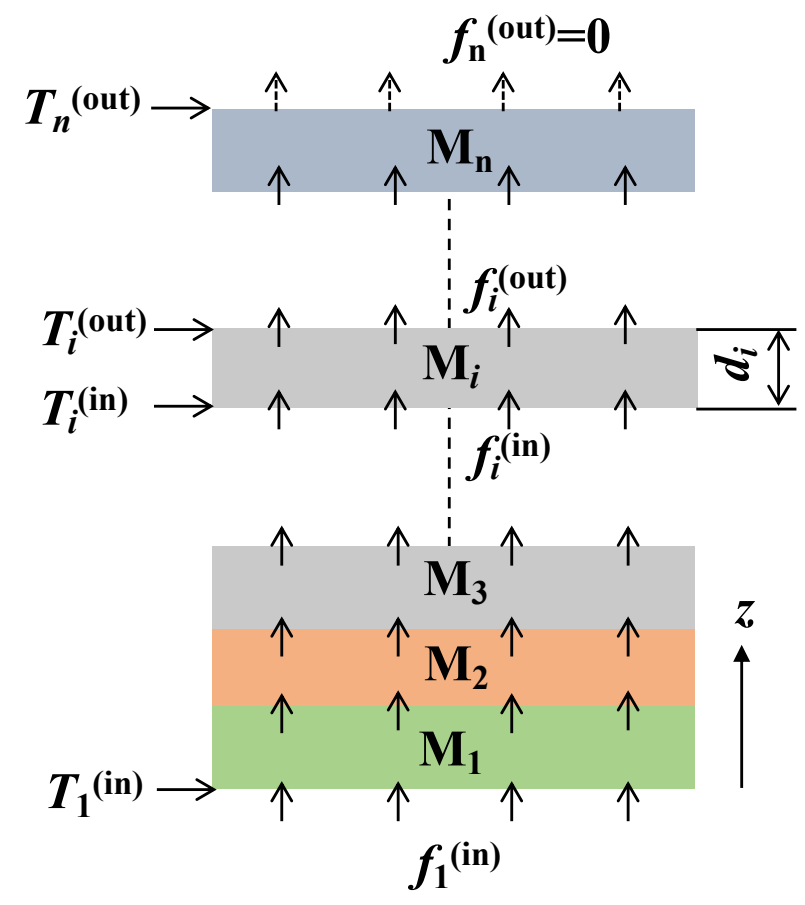

Figure 2: 


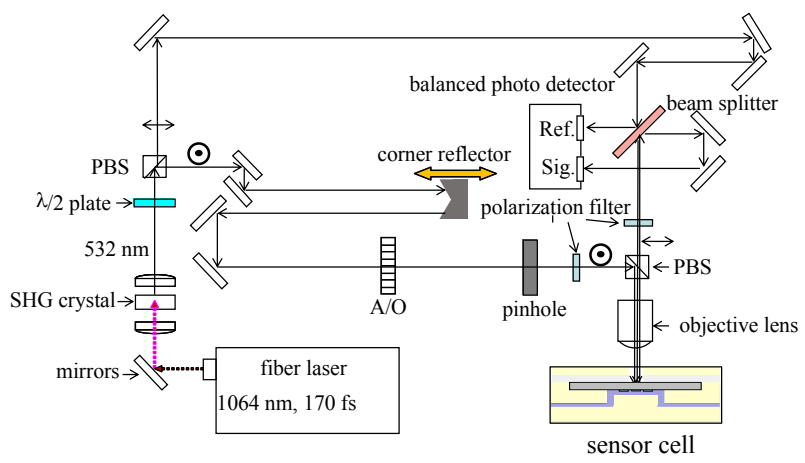

(a)

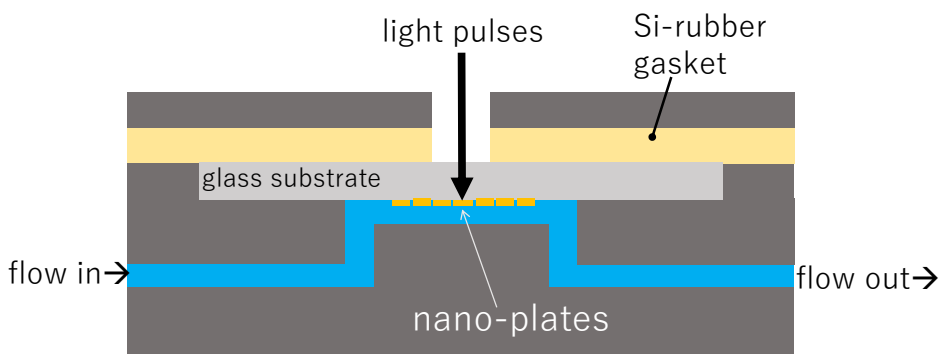

(b)

Figure 3: 

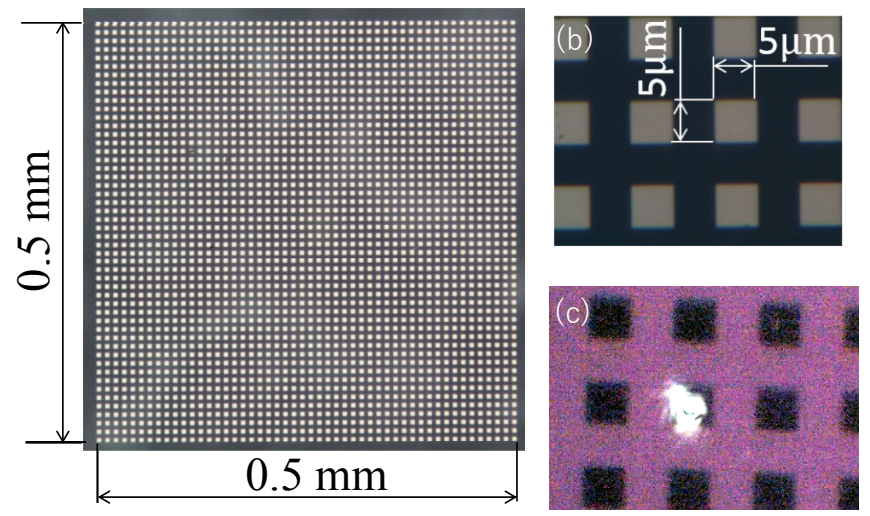

(a)

Figure 4: 

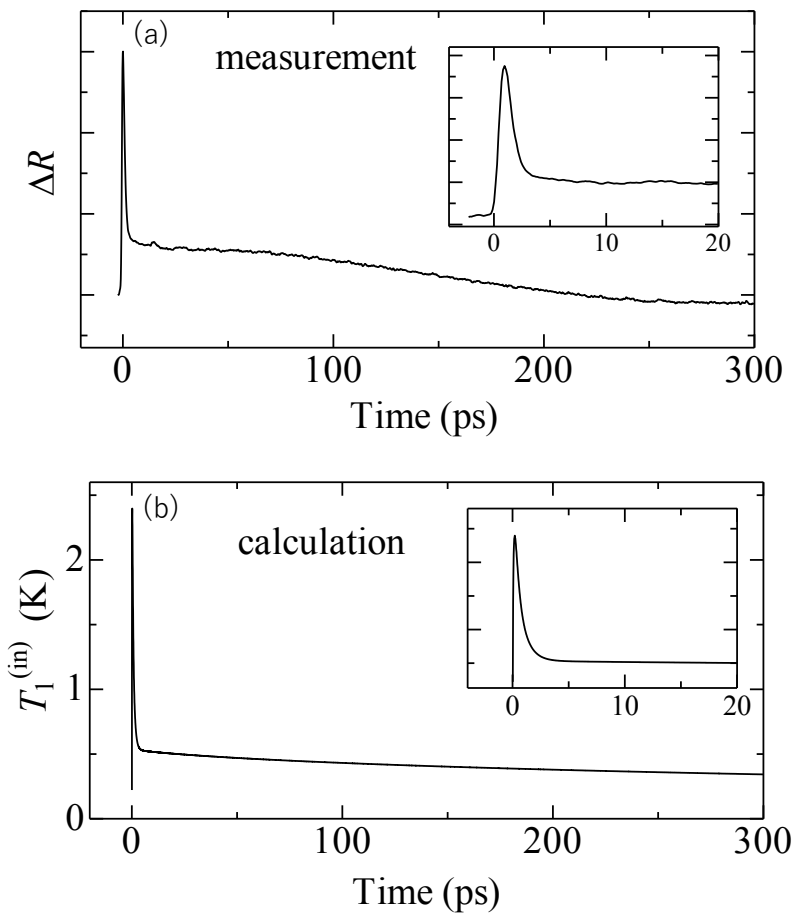

Figure 5: 

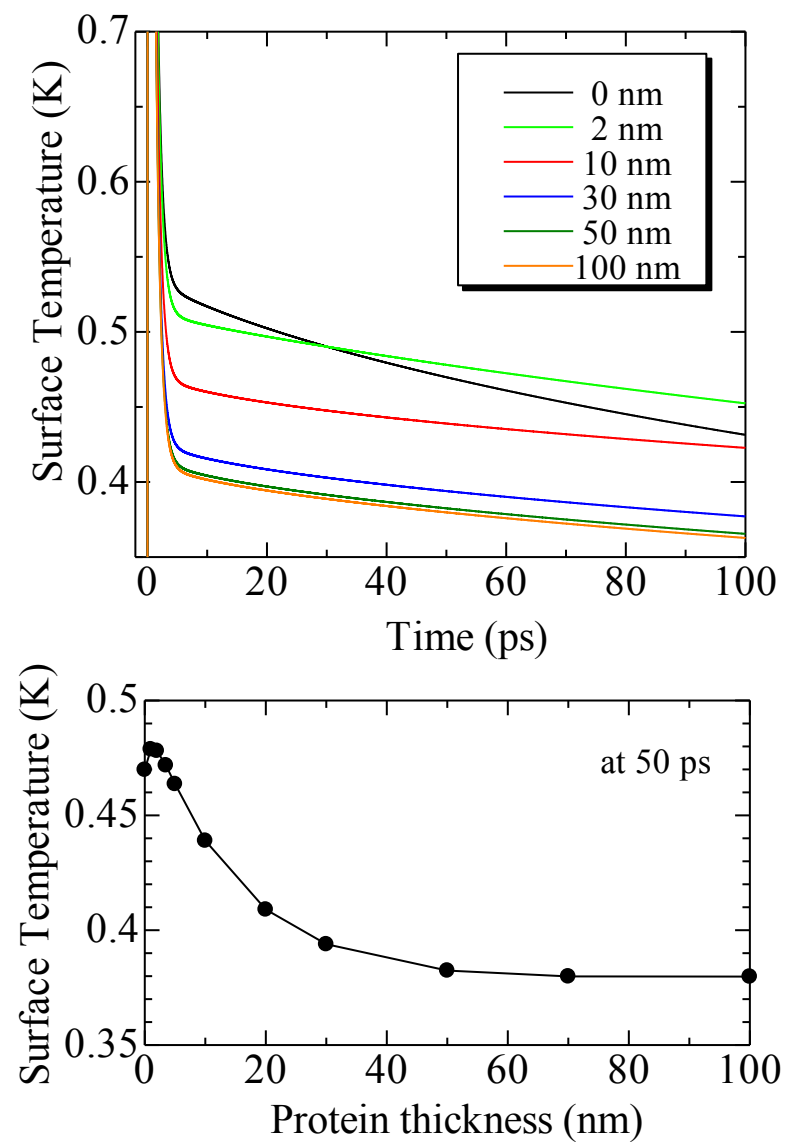

Figure 6: 


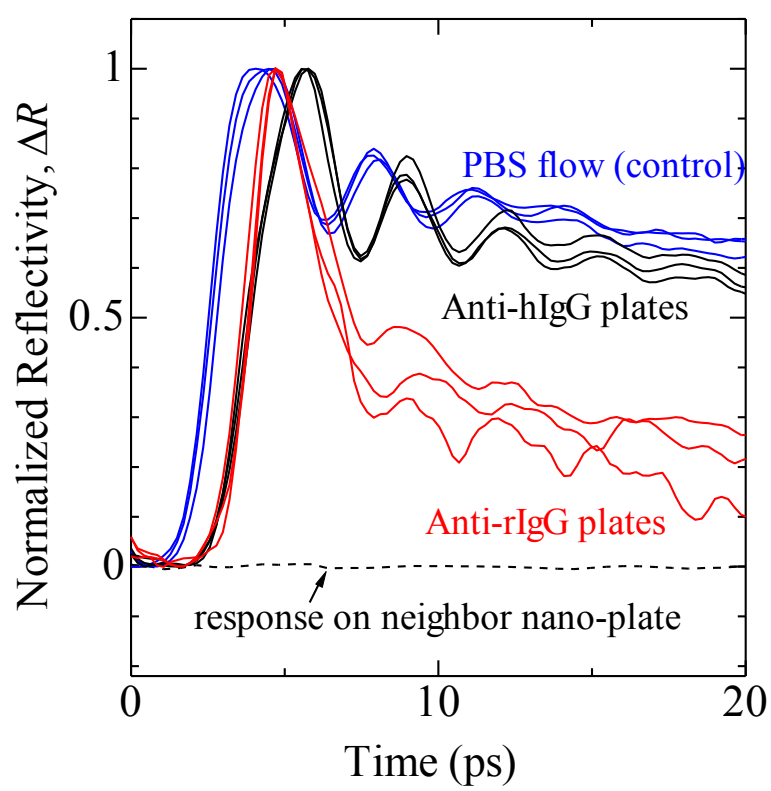

Figure 7: 

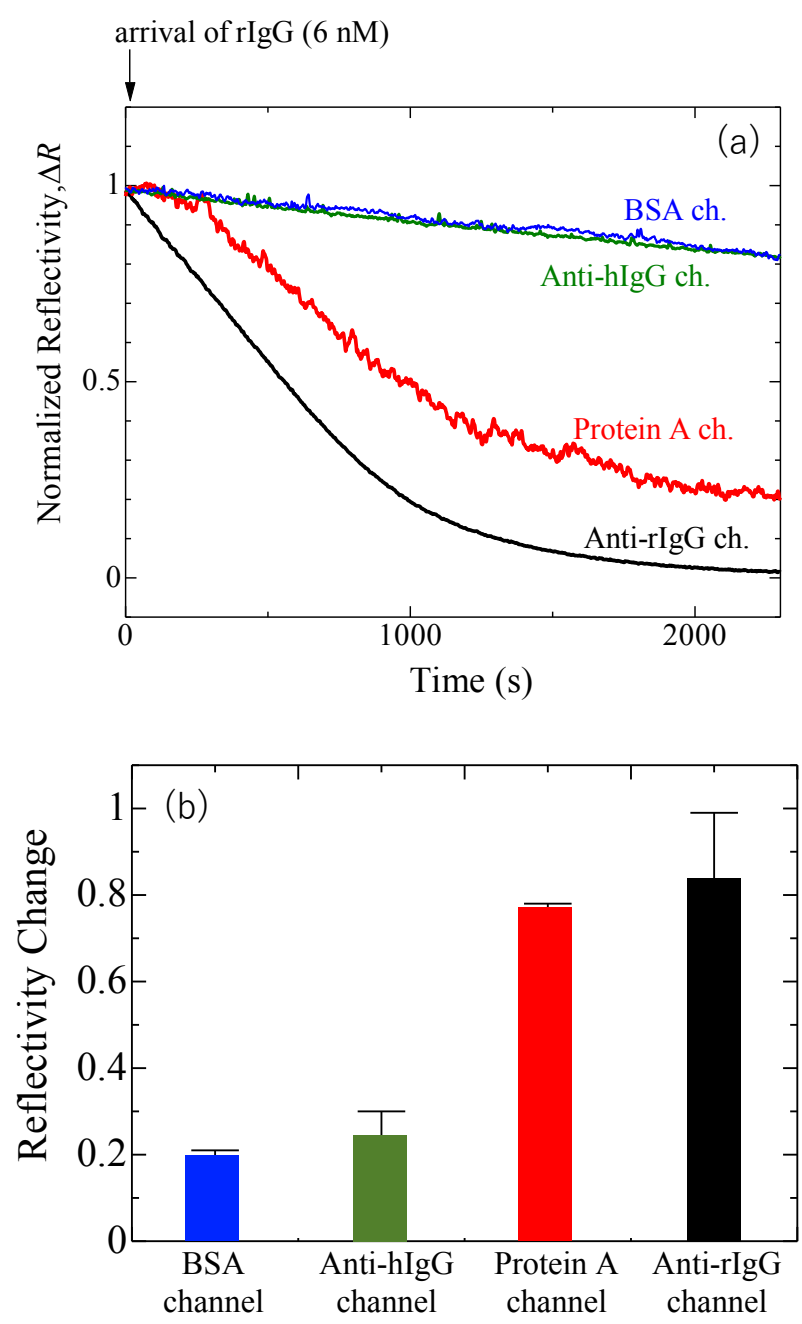

Figure 8: 

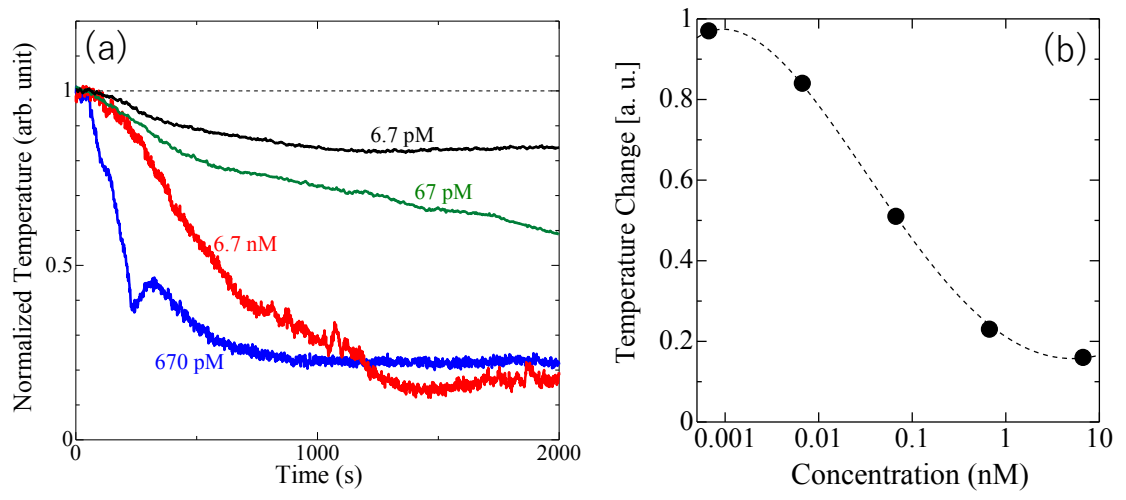

Figure 9: 

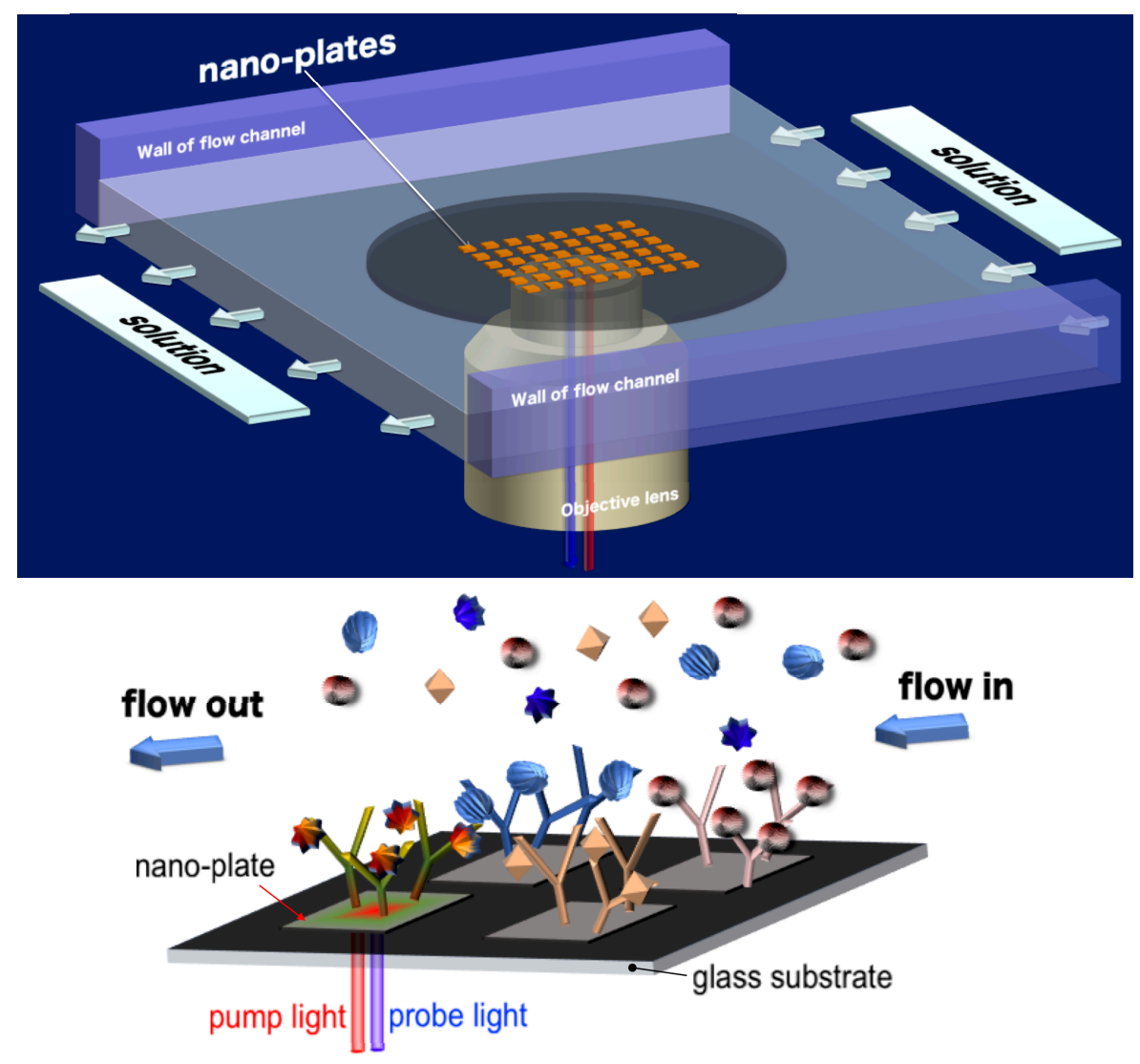

Figure 10: 